\section{PREVALÊNCIA E FATORES ASSOCIADOS À AUTOPERCEPÇÃO NEGATIVA EM SAÚDE DOS ADOLESCENTES: UMA REVISÃO SISTEMÁTICA}

\author{
Prevalence of and factors associated with the adolescents' \\ negative self-perception in health: a systematic review \\ Prevalencia y factores asociados a la autopercepción negativa \\ de salud de adolescentes: una revisión sistemática
}

\section{RESUMO}

Objetivo: Revisar na literatura a prevalência e os fatores associados à autopercepção negativa em saúde dos adolescentes. Métodos: Trata-se de uma revisão sistemática que utilizou, como fonte de busca, as seguintes bases de dados: Lilacs, Medline/Pubmed e Adolec, utilizando descritores indexados ao DeCS/MeSH. No processo de seleção, os artigos foram analisados em três etapas: leitura dos títulos, dos resumos e do artigo na íntegra de acordo com os critérios de elegibilidade. Resultados: Dos 886 artigos encontrados, após a utilização dos filtros, foram selecionados 25 artigos para leitura na integra, mas, após a leitura, somente 10 artigos foram inclusos nos resultados desta pesquisa. Os estudos apresentaram uma prevalência de autopercepção negativa em adolescentes que variou de $1,2 \%$ a $38 \%$, sendo verificados também outros fatores associados, como fatores socioeconômicos, relações interpessoais e comportamentos de riscos à saúde. Conclusão: As evidências científicas apontam que fatores socioeconômicos, relação com a família e amigos, estresse, aspectos psicológicos e comportamentos de risco a saúde estão interligados à autopercepção negativa em saúde dos adolescentes. Além disso, ser do sexo feminino, ter baixa renda e apresentar uma idade maior também foram fatores para um aumento na avaliação negativa do estado de saúde do adolescente.

Descritores: Percepção; Adolescente; Saúde do Adolescente; Fatores de Risco; Autopercepção.

\section{ABSTRACT}

Objective: To review in the literature the prevalence of and the factors associated with the adolescents' negative self- perception in health. Methods: A systematic review that used, as sources for search, the following databases: LILACS, MEDLINE/PubMed and ADOLEC, using descriptors indexed to DeCS/MeSH. In the selection process, the articles were analyzed in three stages: reading of the titles, abstracts and full texts of the articles according to the eligibility criteria. Results: Of the 886 articles found, after the using the filters, 25 articles were selected for full-text reading but, after that, only 10 articles were included in the results of this research. The studies showed a prevalence of negative self-perception in adolescents ranging from $1.2 \%$ to $38 \%$, and other associated factors, such as the socioeconomic factors, interpersonal relationships, and health risk behaviors were also verified. Conclusion: The scientific evidence indicates that socioeconomic factors, relationship with family and friends, stress, psychological aspects and health risk behaviors are linked to the adolescents' negative self-perception in health. Furthermore, being female, having low income, and being older were also factors for an increase in the adolescent's negative evaluation of their health status.

Descriptors: Perception; Adolescent; Adolescent Health; Risk factors; Self Concept.
Artigo de Revisão
Vanessa Mayana Alves Baad ${ }^{(1)}$

Paula Andréa de Melo Valença ${ }^{(1)}$ Valdenice Aparecida Menezes ${ }^{(1)}$

Viviane Colares Amorim ${ }^{(1)}$

Carolina Bandeira Ferreira Santos da Franca ${ }^{(1)}$
(1) Universidade de Pernambuco - UPE Recife (PE) - Brasil
Recebido em: 06/07/2016 Revisado em: 02/08/2016 Aceito em: 09/11/2016 


\section{RESUMEN}

Objetivo: Revisar la prevalencia y los factores asociados a la autopercepción negativa de salud de adolescentes en la literatura. Métodos: Se trata de una revisión sistemática que se utilizó como fuente de búsqueda las siguientes bases de datos: LILACS, MEDLINE/PubMed y Adolec utilizando los descriptores indexados al DeCS/MeSH. En el proceso de selección los artículos fueron analizados en tres etapas: la lectura de los títulos, de los resúmenes y del artículo completo según los criterios de elegibilidad. Resultados: Después de la utilización de los filtros fueron elegidos 25 artículos para la lectura del texto completo de los 886 artículos encontrados, pero tras la lectura, solamente 10 artículos fueron incluidos en los resultados de esa investigación. Los estudios presentaron una prevalencia de autopercepción negativa de adolescentes que varió entre 1,2\% y $38 \%$ y fueron verificados también otros factores asociados como los factores socioeconómicos, las relaciones interpersonales y las conductas de riesgo para la salud. Conclusión: Las evidencias cientificas señalan que los factores socioeconómicos, la relación con la familia y los amigos, el estrés, los aspectos psicológicos y las conductas de riesgo para la salud están asociados a la autopercepción negativa de salud de los adolescentes. Además de eso, pertenecer al sexo femenino, tener baja renta y más edad también fueron factores para un aumento de la evaluación negativa del estado de salud del adolescente.

Descriptores: Percepción; Adolescente; Salud del Adolescente; Factores de Riesgo; Autoimagen.

\section{INTRODUÇÃO}

A autopercepção de saúde se associa fortemente com o estado real ou objetivo de saúde e pode ser encarada como uma representação das avaliações subjetivas de saúde ${ }^{(1)}$, que contemplam aspectos físico, cognitivo e emocional ${ }^{(2)}$. É obtida questionando-se o indivíduo a respeito de como ele classifica a sua saúde nos últimos dias, apresentando como respostas cinco classificações que variam de muito boa a muito ruim ${ }^{(1)}$.

Embora a mensuração do estado geral de saúde dos indivíduos seja bastante complexa, a autopercepção da saúde tem se mostrado um método confiável e mais utilizado do que a observação direta para a análise global da saúde ${ }^{(3,4)}$. Além disso, é um poderoso indicador de mortalidade. Pessoas que relatam a sua saúde como sendo pobre apresentam maior risco de mortalidade por todas as causas de morte em comparação com aquelas que relatam ter uma saúde muito boa ${ }^{(5)}$.

Devido à importante associação entre autopercepção de saúde e mortalidade, a análise dos fatores determinantes de saúde se torna importante, pois modular esses fatores significa alterar a percepção de saúde e pode significar alterar a mortalidade ${ }^{(6)}$. Informações acerca deste indicativo de saúde para a população brasileira demonstraram que a prevalência de saúde negativa em cidades brasileiras variou, sendo de 2,4\% em Palmas (Tocantins), 7,5\% em Macapá (Amapá) e 5,4\% em Salvador (Bahia) ${ }^{(7)}$.

Em um inquérito realizado pelo sistema de vigilância de fatores de risco e proteção nacional, foram apresentados dados das 27 capitais do Brasil e do Distrito Federal sobre a autopercepção em saúde da população adulta e idosa ${ }^{(8)}$. Porém, para adolescentes brasileiros, essas informações ainda são escassas ${ }^{(9)}$, existindo alguns estudos no cenário nacional ${ }^{(9-11)} \mathrm{e}$ internacional ${ }^{(12-13)}$.

A adolescência é considerada uma categoria sociocultural, de origem histórica, que envolve dimensões biopsicológicas e sociais $^{(14,15)}$. É um período de rápidas mudanças físicas e psicossociais, em que o adolescente passa por um processo de descobrir como viver a vida, os modos de ser e estar com os outros, até a construção do futuro com as escolhas profissionais ${ }^{(15)}$.

É importante ressaltar que a adolescência é um período crucial para adoção de novos estilos de vida e percepções de saúde ${ }^{(16)}$, e estudos sobre os determinantes da autopercepção da saúde do adolescente são limitados ${ }^{(9,11,17)}$. Frente ao exposto, o presente estudo tem como objetivo revisar na literatura a prevalência e os fatores associados à autopercepção negativa em saúde dos adolescentes.

\section{MÉTODOS}

Trata-se de uma revisão sistemática, conduzida no período de outubro de 2015 a março de 2016, com o objetivo de realizar uma síntese de artigos que analisaram a prevalência e os fatores associados à autopercepção negativa de saúde em adolescentes.

A pesquisa foi realizada nas seguintes bases de dados eletrônicas: Lilacs (Literatura Latino-Americana e do Caribe em Ciências da Saúde), ADOLEC e Medline/ Pubmed (National Library of Medicine National Institutes of Health).

Como ponto de partida, foi realizado a seleção dos descritores mediante a consulta ao DeCS (Descritores em Ciências da Saúde) e ao MeSH (Medical Subject Headings), sendo considerados os seguintes descritores na língua portuguesa e inglesa respectivamente: "Percepção de Saúde", "Adolescente", "Saúde do adolescente", "Fatores de Risco" e "Percepção negativa em Saúde" ("Health Perception," "Adolescent", "Adolescent health”, "Risk Factors", "Negative Self Perception") além do operador lógico "and" para combinação dos termos. Os descritores foram combinados por pares e, em seguida, por conjunto para se ter uma melhor captação dos artigos. 
Todo o processo de busca, seleção e avaliação de artigos foi realizado por dois pesquisadores de maneira independente, em que as publicações que preencheram os critérios de elegibilidade foram analisadas integralmente e independentemente por dois pesquisadores. Em seguida, as discordâncias foram confrontadas a fim de se obter um consenso entre os pares.

Os critérios de elegibilidade do estudo foram: artigos publicados nos idiomas inglês, português ou espanhol; publicados entre os anos de 2010 a 2016, tendo como população indivíduos adolescentes, e estudos que tratassem da autopercepção negativa da saúde do adolescente. Foram excluídos os artigos de revisão, teses, dissertações e monografias, estudos duplicados em mais de uma base de dados e aqueles artigos que não avaliaram a autopercepção de saúde negativa em adolescentes.
O processo de seleção dos artigos ocorreu por meio da leitura do título, seguido do resumo e texto completo, respectivamente. Os artigos selecionados foram avaliados em relação ao dimensionamento da amostra, pois se considerou que essa etapa metodológica era fundamental para a obtenção de uma resposta válida para a pergunta dessa revisão.

\section{RESULTADO}

Entre os principais resultados encontrados verificou-se que a autopercepção de saúde negativa esteve associada à fatores socioeconômicos ${ }^{(9,11,17)}$ e ambientais ${ }^{(11)}$, assim como à condutas comportamentais ${ }^{(18-20)}$.

Os estudos apresentaram também associação com o hábito de fumar ${ }^{(9,19,20)}$, baixo índice de atividade

Quadro I - Características dos artigos relacionados à autopercepção de saúde em adolescentes entre os anos de 2010 a 2016.

\begin{tabular}{|c|c|c|c|c|}
\hline Autor (Ano) & $\begin{array}{l}\text { Local do } \\
\text { Estudo }\end{array}$ & $\begin{array}{l}\text { Número de } \\
\text { Participantes }\end{array}$ & Faixa Etária & Principais associações aos fatores de riscos \\
\hline Sousa et al. $(2010)^{(9)}$ & Brasil & 5028 & 15 a 19 anos & $\begin{array}{l}\text { Sexo feminino, baixa condição econômica, uso } \\
\text { de tabaco e alto nível de estresse. }\end{array}$ \\
\hline Meireles et al. $(2015)^{(11)}$ & Brasil & 1042 & 11 a 17 anos & $\begin{array}{l}\text { Sexo, fatores socioambientais, problemas } \\
\text { familiares, com pares e vizinhança. }\end{array}$ \\
\hline Spein et al. $(2013)^{(17)}$ & Noruega & 728 & 15 e 16 anos & $\begin{array}{l}\text { Fatores socioeconômicos, suicídio e baixo nível } \\
\text { de atividade física. }\end{array}$ \\
\hline Mendonça et al. $(2012)^{(18)}$ & Brasil & 2859 & 14 a 19 anos & $\begin{array}{l}\text { Fatores socioeconômicos, baixo nível de } \\
\text { atividade física e excesso de peso corporal. }\end{array}$ \\
\hline Afridi et al. $(2013)^{(19)}$ & Paquistão & 414 & 14 a 17 anos & $\begin{array}{l}\text { Sexo masculino, qualificação dos pais, } \\
\text { problemas familiares, estilo de vida, uso de } \\
\text { tabaco e dieta pouco saudável. }\end{array}$ \\
\hline Richter et al. $(2012)^{(20)}$ & Alemanha & 6997 & 11 a 15 anos & $\begin{array}{l}\text { Sexo feminino, baixa condição econômica, má } \\
\text { estrutura familiar, dieta pobre, uso de tabaco, e } \\
\text { baixo nível de atividade física. }\end{array}$ \\
\hline Farias Júnior et al. (2012) $)^{(21)}$ & Brasil & 2874 & 14 a 19 anos & Sexo feminino, baixo nível de atividade física. \\
\hline Galán et al. $(2016)^{(22)}$ & Espanha & 21811 & 11 a 18 anos & $\begin{array}{l}\text { Baixo nível de atividade física, menos satisfação } \\
\text { com a vida. }\end{array}$ \\
\hline Wiklund et al. $(2012)^{(23)}$ & Suécia & 1027 & 16 a 18 anos & Sexo feminino e estresse. \\
\hline Meireles et al. $(2015)^{(24)}$ & Brasil & 1035 & 11 a 17 anos & $\begin{array}{l}\text { Estrutura familiar, baixo consumo de frutas e } \\
\text { verduras, sobrepeso, baixo peso e insatisfação } \\
\text { com a vida. }\end{array}$ \\
\hline
\end{tabular}


física $^{(11,17,21,22)}$, estresse ${ }^{(9,23)}$, insatisfação com o peso e com a $\operatorname{vida}^{(24)}$, e comportamentos associados ao suicídio ${ }^{(17)}$ (Quadro I).

Dos estudos incluídos, todos apresentaram em sua amostra adolescentes, variando a idade entre 11 aos 19 anos. O tamanho amostral dos estudos variou entre $414^{(19)}$ a $21.811^{(22)}$ adolescentes. Todos os estudos fizeram a opção por utilizar questionários validados, assim como utilizaram o método transversal para o delineamento do estudo.

Em relação à prevalência total da autopercepção negativa em saúde, os percentuais variaram desde $1,2 \%{ }^{(11)}$, mínima apontada, até $38 \%{ }^{(17)}$, para a porcentagem máxima encontrada nos estudos.

Dos estudos selecionados, três ${ }^{(9,18,21)}$ se encontram disponíveis no idioma português e os demais na língua inglesa. A prevalência da autopercepção negativa quando comparada ao sexo variou de $6,7 \%^{(19)}$ a $32,2 \%^{(22)}$ para o sexo masculino e de $11 \%{ }^{(24)}$ a $53,6 \%^{(11)}$ para o sexo feminino (Quadro II).

\section{DISCUSSÃO}

Através dos dados encontrados nesta revisão, foi possível encontrar informações acerca da autopercepção em saúde como: i) A metodologia utilizada para os estudos em relação ao delineamento estão associadas aos estudos transversais; ii) A utilização de questionários validados foi a forma mais comum para avaliar a autopercepção em saúde dos adolescentes; iii) Os resultados encontrados em questão da prevalência da autopercepção negativa são representativos para as associações feitas nos estudos; iv) Maior prevalência de autopercepção negativa nas mulheres; v) Quando avaliados os fatores associados, os dados socioeconômicos, baixo nível de atividade física, relações entre família e escola e atitudes comportamentais de risco à saúde estão relacionados à autopercepção negativa em adolescentes.

Inicialmente, a pergunta utilizada para avaliação da autopercepção em saúde foi elaborada com essência igualitária por todos os estudos, havendo uma pergunta direta com resposta pessoal, a qual é avaliada nos estudos por quatro ${ }^{(19,20,22,23)}$ ou $\operatorname{cinco}^{(9,11,17,18,21,24)}$ alternativas que variam de muito boa a muito ruim.

Todos os estudos fizeram o uso de questionário para avaliar a autopercepção negativa em saúde e seus fatores associados, apresentando uma variância de instrumentos. Foram utilizados questionários como o PeNSE(11), $\mathrm{COMPAQ}^{(9)}$ e o WBYG ${ }^{(17)}$, o que pode justificar a variação nas prevalências obtidas quanto as associações, uma vez que os questionários são escolhidos para melhor atender os objetivos do estudo.
A autopercepção negativa em saúde é uma variável bastante utilizada para estudos epidemiológicos e merece uma atenção especial para o período da adolescência, em que existem diversas alterações físicas, psicológicas e comportamentais, tendo uma prevalência que varia entre de $1,2 \%^{(11)}$ a $38 \%{ }^{(17)}$ nessa faixa etária.

Um fator importante a ser observado é que, nos achados dos autores quanto aos aspectos socioeconômicos, evidenciou-se que adolescentes com menor renda familiar, apresentam uma maior chance de apresentar uma autopercepção negativa em saúde $\mathrm{e}^{(9,11,18,20,24)}$. Sabe-se que a renda representa um componente determinante no maior acesso às atividades de lazer, educação, moradia e serviços de saúde ${ }^{(9)}$.

A percepção negativa de saúde também esteve associada quando comparada aos problemas familiares ${ }^{(19,20,24)}$, tais como distanciamento familiar ${ }^{(19)}$, apoio nas decisões e estrutura familiar ${ }^{(20,24)}$. Geralmente, os adolescentes que vivem em famílias em que há um maior apoio social presente, encontram-se numa melhor autopercepção de saúde ${ }^{(27)}$.

Em relação ao sexo, houve uma prevalência mais elevada para as adolescentes do sexo feminino na grande maioria dos estudos ${ }^{(09,11,17,18,20-22,24)}$. Apenas dois estudos apresentaram uma maior percepção negativa para adolescentes do sexo masculino ${ }^{(19,23)}$. Acredita-se que, culturalmente, as moças façam mais exames de rotina e visitem com maior frequência um médico, o que aumenta a chance de diagnóstico precoce de alguma doença. Além disso, elas apresentam maior sensibilidade para detectar alterações fisiológicas ${ }^{(28)}$ e considerar hábitos inadequados para a saúde ${ }^{(29)}$.

Um estudo(11) afirma que a autopercepção negativa em saúde esteve associada ao aumento da idade dos adolescentes, sugerindo que os adolescentes podem se tornar cada vez mais preocupados com sua saúde no passar dos anos assim como podem adotar outros comportamentos de riscos com o decorrer do tempo. Esses resultados corroboram com os achados de outra pesquisa ${ }^{(18)}$, a qual demonstrou que adolescentes mais velhos apresentam uma pior percepção de saúde, principalmente adolescentes do sexo feminino.

Outra variável que apresentou resultados importantes foi a relação com a família e amigos, sendo verificado que adolescentes que têm uma má relação com os pais e/ou amigos apresentam resultados superiores na autopercepção negativa em saúde ${ }^{(11,19,20)}$, o que demonstra a importância do círculo de convivência dos adolescentes com seus pares e o quanto isso influência na sua percepção de saúde.

Quanto a associação com comportamentos de risco à saúde, um estudo ${ }^{(9)}$ apresentou que adolescentes que 
Quadro II - Avaliação da saúde negativa dos adolescentes, estratificando-se por gênero e periódico publicado.

\begin{tabular}{|c|c|c|c|c|}
\hline \multirow{2}{*}{ Autor (Ano) } & \multirow{2}{*}{$\begin{array}{l}\text { \% Saúde } \\
\text { negativa }\end{array}$} & \multirow{2}{*}{ Periódico } & \multicolumn{2}{|c|}{ Prevalência de sexo (\%) } \\
\hline & & & (Masculino) & (Feminino) \\
\hline Sousa et al. $(2010)^{(9)}$ & $14,4 \%$ & Rev. Paulista de Pediatria & $9,6 \%$ & $17,6 \%$ \\
\hline Meireles et al. $(2015)^{(11)}$ & $1,2 \%$ & $\begin{array}{l}\text { Rev. Brasileira de } \\
\text { Epidemiologia }\end{array}$ & $46,6 \%$ & $53,6 \%$ \\
\hline Spein et al. $(2013)^{(17)}$ & $38 \% * 11 \% * *$ & $\begin{array}{c}\text { Int. Journal of Circumpolar } \\
\text { Health }\end{array}$ & $9 \% * 28 \% * *$ & $11 \% * 41 \% * *$ \\
\hline Mendonça et al. $(2012)^{(18)}$ & $15,8 \%$ & $\begin{array}{c}\text { Rev. Bras. Atividade Física e } \\
\text { Saúde }\end{array}$ & $10,3 \%$ & $19,9 \%$ \\
\hline Afridi et al. $(2013)^{(19)}$ & $29 \%$ & $\begin{array}{l}\text { Global Journal of Health } \\
\text { Science }\end{array}$ & $32,2 \%$ & $23,6 \%$ \\
\hline Richter et al. $(2012)^{(20)}$ & $13,9 \%$ & J. Epidemiol Community Health & $12 \%$ & $16 \%$ \\
\hline Farias Júnior et al. $(2012)^{(21)}$ & $15,8 \%$ & Rev. de Saúde Pública & $10,3 \%$ & $19,9 \%$ \\
\hline Galán et al. (2016) & $8,9 \%$ & BMJ Open & $6,7 \%$ & $10,7 \%$ \\
\hline Wiklund et al. (2012) & $16,4 \%$ & BMC Public Health & $20.5 \%$ & $12,2 \%$ \\
\hline Meireles et al. $(2015)^{(24)}$ & $11,2 \%$ & PLoS One & $\begin{array}{c}8,3 \%^{\mathrm{a}} \\
10,9 \%^{\mathrm{b}}\end{array}$ & $\begin{array}{l}6,9 \%^{\mathrm{a}} \\
16,9 \%^{\mathrm{b}}\end{array}$ \\
\hline
\end{tabular}

* Refere-se à amostra da população de Inuit; ** Refere-se à amostra da população de Sami.

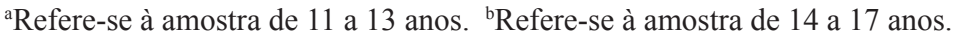

alegaram fumar têm uma maior prevalência de saúde negativa e esse mesmo resultado esteve presente em outros estudos $^{(25,26)}$. A associação relacionada ao uso de tabaco também esteve presente em outras pesquisas ${ }^{(19,20)}$.

Outra pesquisa ${ }^{(31)}$ também visou analisar a influência de alguns fatores em associação com a percepção de saúde, sendo identificada que, das três drogas investigadas (álcool, tabaco e maconha), apenas o tabaco apareceu como preditor de percepção negativa de saúde.

Esta revisão não apresentou achados importantes quanto ao consumo de álcool e percepção negativa em saúde, entretanto, a literatura aponta que adolescentes que optaram em não responder a questão tiveram uma probabilidade maior para a saúde negativa quando comparados aos que responderam ${ }^{(9)}$.

O baixo nível de atividade física também foi apresentado como indicador para a autopercepção negativa em saúde ${ }^{(9,17,18,20-22)}$. Esses dados foram iguais a um estudo de base em saúde que incluiu 2741 adolescentes com idade entre 13 e 19 anos, sendo observado que a ausência de exercício físico é um fator de predisposição para a avaliação negativa em saúde ${ }^{(27)}$. Adolescentes fisicamente ativos se mostraram mais propensos a perceber seu estado de saúde de forma positiva ${ }^{(32)}$.

Dieta pouco saudável também esteve associada à percepção negativa de saúde nos adolescentes ${ }^{(10,18,19,24)}$. Um estudo realizado pelo Centro Norte-Americano de Controle e Prevenção de Doenças (CDC), descobriu que menos de um quarto dos adolescentes comem bastante frutas e legumes ${ }^{(33)}$. O sobrepeso também esteve presente em alguns estudos $^{(18,24)}$, tendo associação direta com a percepção de saúde dos adolescentes. Isso alerta a preocupação com a alimentação dos adolescentes, apontando a necessidade de uma dieta balanceada para se ter uma regularidade no peso corporal, obtendo-se aumento dos índices positivos relacionados à percepção de saúde.

O estresse também esteve associado à percepção negativa em adolescentes ${ }^{(23)}$. Um estudo de 2012 $2^{(23)}$ evidenciou que adolescentes do sexo feminino têm um índice mais elevado de estresse comparado aos adolescentes do sexo masculino. Resultados similares também foram encontrados em estudo anterior de $2010^{(9)}$. Sabidamente, essa é uma fase de formação e inserção do indivíduo em situações diferenciadas, como o mercado de trabalho e 
estudos, além do aumento das responsabilidades pessoais o que pode ocasionar um elevado índice de estresse.

Outra variável que também chamou atenção foi a insatisfação com a vida ${ }^{(22,25)}$. A importância do bem-estar psicológico, no que diz respeito à percepção de saúde dos adolescentes, tem sido observado por estudos que têm abordado a saúde subjetiva avaliada através de uma medida de um único item ${ }^{(34,35)}$, o qual visa perceber o nível de satisfação com a vida dos adolescentes.

As diferenças entre os estudos que analisam a percepção de saúde em adolescentes podem ser ocasionadas pela ausência de uma norma internacional para as opções de resposta ou pela maneira em que a variável é categorizada. Essa diferença de resultados pode estar relacionada também à localização do adolescente, ao local de preenchimento do questionário, ao tipo de questionário e à forma de escolha do indivíduo que irá ao responder estudo. Ressalta-se a importância de estudos em que esses dados auxiliam o âmbito de intervenções em saúde pública, através da elaboração de estratégias de promoção de hábitos saudáveis, a fim de atingir o público adolescente, dando ênfase na mudança de comportamento para uma alteração dos fatores de risco, para que o adolescente possa responder de forma positiva à percepção de saúde.

\section{CONCLUSÃO}

As evidências científicas apontam que fatores socioeconômicos, relação com famílias e amigos, estresse, aspectos psicológicos e comportamentos de risco à saúde estão interligados à autopercepção negativa em saúde de adolescentes. Além disso, ser do sexo feminino, ter baixa renda, e apresentar uma idade maior também foram fatores para um aumento na avaliação negativa do estado de saúde do adolescente.

\section{REFERÊNCIAS}

1. Appels A, Bosma H, Grabauskas V, Gostautas A, Sturmans F. Self-rated health and mortality in a Lithuanian and a Dutch population. Soc Sci Med. 1996;42(5):681-9.

2. Ofstedal MB, Zimmer Z, Cruz G, Chan A, Lin YH. Self-assessed health expectancy among older Asians: a comparison of Sullivan and multistate life table methods. Ann Arbor: Michigan Center on the Demography of Aging; 2016. (Elderly in Asia Report n. 03-60).

3. Bailis DS, Segalla A, Chipperfield JG. Two view of self- rated general health status. Soc Sci Med. 2003;56(2):203-17.
4. Martikanen P, Aroma A, Heliovaara M, Klaukka T, Knekt P, Maatela J, et al. Reliability of perceived health by sex and age. Soc Sci Med. 1999;48(8):1117-22.

5. Marcellini F, Leonardi F, Marcucci A, Freddi A. Health perception of elderly people: the results of a longitudinal study. Arch Gerontol Geriatr Suppl. 2002;8:181-9.

6. Carneiro-Campos LE, Fernandes CP, Balduíno A, Leite Duarte ME, Leitão M. The effect of titanium topography features on mesenchymal human stromal cells' adhesion. Clin Oral Implants Res. 2010;21(2):250-4.

7. Ministério da Saúde (BR). Vigilância de fatores de risco e proteção para doenças crônicas por inquérito telefônico: estimativas sobre freqüência e distribuição sócio-demográfica de fatores de risco e proteção para doenças crônicas nas capitais dos 26 Estados brasileiros e no Distrito Federal em 2006. Brasília: Ministério da Saúde; 2009.

8. Conselho Nacional de Secretários de Saúde (BR). Regulação em saúde. Brasília: CONASS; 2011.

9. Sousa TFS, Silva KS, Garcia LMT, Del Duca GF, Oliveira ESA, Nahas MV. Autoavalição de saúde e fatores associados em adolescentes do Estado de Santa Catarina, Brasil. Rev Paul Pediatr. 2010;28(4):333-9.

10. Reichert FF, Loch MR, Capilheira MF. Autopercepção de saúde em adolescentes, adultos e idosos. Ciênc Saúde Coletiva. 2012;17(12):3353-62.

11. Meireles AL, Xavier CC, Proietti FA, Caiaffa WT. Influence of individual and socio-environmental factors on self-rated health in adolescents. Rev Bras Epidemiol. 2015;18(3):538-51.

12. Larson JS. The World Health Organization's definition of health: Social versus spiritual health. Soc Indic Res, 1996;38(2):181-92.

13. Breidablik HJ, Meland E, Lydersen S. Self-rated health in adolescence: a multifactorial composite. Scand J Public Health 2008;36(1):12-20.

14. Peres F, Rosenburg CP. Desvelando a concepção de adolescência/adolescente presente no discurso da saúde pública. Saúde Soc. 1998;7(1):53-86

15. Ferreira MA, Alvim NAT, Teixeira MLO, Veloso RC. Saberes de adolescentes: estilo de vida e cuidado à saúde. Texto \& Contexto Enferm. 2007;16(2):217-24.

16. Loch MR, Possamai CL. Associação entre percepção de saúde e comportamentos Relacionados à saúde em adolescentes escolares de Florianópolis-SC. Ciênc Cuid Saúde. 2007;6:377-83. 
17. Spein AR, Pedersen CP, Silviken AC, Melhus M, Kvernmo SE, Bjerregaard P. Self-rated health among Greenlandic Inuit and Norwegian Sami adolescents: associated risk and protective correlates. Int $\mathrm{J}$ Circumpolar Health. 2013;72:19793.

18. Mendonça G, Cazuza JFJ. Percepção de saúde e fatores associados em adolescentes. Rev Bras Ativ Fís Saúde. 2012;17(3):174-80.

19. Afridi AAK, Motwani K, Khawaja S, Khoja AA, Fatmi Z, Azam I, et al. Self-perceived health among school going adolescents in Pakistan: influence of individual, parental and life style factors? Glob J Health Sci. 2013;5(4):71-8.

20. Richter M, Moor I, van Lenthe FJ. Explaining socioeconomic differences in adolescent self-rated health: the contribution of material, psychosocial and behavioural factors. J Epidemiol Community Health 2012;66(8):691-7.

21. Farias JC Júnior, Lopes ADS, Mota J, Hallal PC. Prática de atividade física e fatores associados em adolescentes no Nordeste do Brasil. Rev Saúde Publica. 2012;46(3):505-15.

22. Galan I, Boix R, Medrano MJ, Ramos P, Rivera F, Pastor-Barriuso R, et al. Physical activity and selfreported health status among adolescents: a crosssectional population-based study. BMJ Open.2013;3:110.

23. Wiklund M, Malmgren-Olsson EB, Ohman A, Bergström E, Fjellman-Wiklund A. Subjective health complaints in older adolescents are related to perceived stress, anxiety and gender - a cross-sectional school study in Northern Sweden. BMC Public Health.2012;12:993.

24. Meireles AL, Xavier CC, De Souza Andrade AC, Proietti FA, Caiaffa WT. Self-rated health among urban adolescents: the roles of age, gender, and their associated factors. PLoS One. 2015;10(7):1-14.

25. Wade TJ, Vingilis E. The development of self-rated health during adolescence: an exploration of inter and intra-cohort effects. Can J Public Health. 1999; 90(2):90-4.
26. Vingilis ER, Wade TJ, Seeley JS. Predictors of adolescent self-rated health. Analysis of the National Population Health Survey. Can J Public Health. 2002;93(3):193-7.

27. Breidablik HJ, Meland E, Lydersen S. Self-rated health in adolescence: a multifactorial composite. Scand J Public Health. 2008;36(1):12-20.

28. Shadbolt B. Some correlates of self-rated health for Australian women. Am J Public Health. 1997;87(6): 951-6.

29. Meurer LN, Layde PM, Guse CE. Self-rated health status: a new vital sign for primary care? WMJ. 2001;100(7):35-9.

30. Heard HE, Gorman BK, Kapinus CA. Family structure and self-rated health in adolescence and young adulthood. Popul Res Policy Rev. 2008;27:773.

31. Vingilis E, Wade TJ, Adlaf E. What factors predict student self-rated physical health? J Adolesc. 1998;21(1):83-97.

32. Aarnio M, Winter T, Kujala U, Kaprio J. Associations of health related behaviour, social relationships, and health status with persistent physical activity and inactivity: a study of Finnish adolescent twins. Br J Sports Med. 2002;36(5):360-4.

33. Centers for Disease Control and Prevention - CDC. Key strategies for schools to prevent obesity [acesso em 2016 Mar 15]. Disponível em: http://www.cdc.gov/ Features/ChildhoodObesity

34. Karademas EC, Peppa N, Fotiou A, Kokkevi A. Family, school and health in children and adolescents: findings from the 2006 HBSC study in Greece. J Health Psychol. 2008;13(8):1012-20.

35. Mechanic D, Hansell S. Adolescent competence, psychological well-being, and self-assessed physical health. J Health Soc Behav. 1987;28(4):364-74.

\section{Endereço para correspondência:}

Bruno Vieira Souza Silva

Avenida: Professor Moraes Rego, 1235

Bairro: Cidade Universitária

CEP 50670-901 - Recife - PE - Brasil

E-mail: brunorafael45@hotmail.com 